\title{
_Book Review
}

\section{MAGNETIC MATERIALS IN JAPAN: RESEARCH, APPLICATION AND POTENTIAL}

Elsevier Advanced Technology, Elsevier Science Publishers Ltd., Oxford, 1991, $£ 185.00$ (soft cover).

This publication examines the most recent developments in magnetic materials in Japan and predicts the likely courses for research. Japan has become the most important player in research and commercialization of magnetic materials and a leading force in outlining the future course of the magnetics-related research.

Following an introduction, there are five chapters, each written by an acknowledged Japanese authority in the topic. Y. Nakamura and S. Iwasaki (Tohoku University) are the authors of the chapter on perpendicular magnetic recording, a method which is capable of providing very high recording density. This technique permits the recording of 1 bit of information in an area as small as 50x50 $\mathrm{nm}$ which is equivalent to several crystalline grains in a recording medium. Consequently, information stored in 5000 floppy disks could be stored in an area of $1 \mathrm{~cm}^{2}$ of the recording medium.

As a result of rapid development of semiconductor memories, predictions have been made that magnetic recording will be replaced in the future. The authors show that this is not a likely scenario: magnetic recording is reliable, superior in durability, easy-to-use and ultra-large memory capacities can be achieved. 
Remarkable progress has recently been achieved in Japan in improving the manufacturing processes, as well as in magnetic properties of oriented electrical steels and ultra-rapidly quenched silicon steels. Amorphous magnetic materials, with a lower iron loss have emerged as new, powerful rivals to conventional silicon steel sheets. Under these circumstances, efforts have been made to increase the effective level of magnetic flux density of silicon steel sheets and to decrease the level of the iron loss of the materials.

The article written by K. Narita (Chubu University) describes the research and development trends and search for low iron-loss, non-grain-oriented silicon steel sheets, high grain-oriented silicon steel sheets and high-silicon steel sheets.

Production of both soft and hard ferrites in Japan has been rising each year. Ferrite-related research disclosures are, however, rather limited, and the article by K. Okutani (TDK Corp.) on soft ferrite materials and their applications reviews the current technological and market trends. It outlines the trends to develop new high-value-added products such as materials for high- frequency usage, chip coils and filters, magnetooptical applications, noise filters and components for floppy disks and hard disks.

Of great technological and market importance is the development and manufacture of rare-earth permanent magnets. S. Hirosawa (Sumitomo Special Metals Co. Ltd.) describes, in his excellent paper, the current status of development of $\mathrm{NdFeB}$ permanent magnets with special attention paid to differences in four existing production routes, namely powder metallurgical method, melt-spinning method, mechanical alloying method and hot deformation of cast ingots technique.

The problems that the $\mathrm{NdFeB}$ magnets face, viz. thermal and environmental stability, are discussed from the first principles and technological solutions are offered. Future trends in research and development are outlined and it is shown 
that the cost of raw materials, as well as the processing cost must be reduced, for a wider application of $\mathrm{NdFeB}$ magnets to materialize.

This is in contrast to frequent claims that the Nd-based permanent magnets will be cheaper than SmCo magnets because $\mathrm{Nd}$ is six times more abundant than $\mathrm{Sm}$. The author shows that raw material portion of the entire production cost is small and, therefore, the reduction of processing cost is essential.

The current status of development and production of Sm- based permanent magnets is covered in a paper by $\mathrm{H}$. Harada and $\mathrm{M}$. Tokunaga (Hitachi Metals, Ltd.). The advantages of SmCo magnets, in comparison to NdFeB magnets are stressed and efforts to reduce the disadvantages are discussed.

Composition and heat treatment dependency of physical properties of SmCo magnets are analysed in detail and the role of replacement of Sm with other rareearth elements is outlined.

This well-produced publication provides a wealth of information to anyone involved with magnetic materials. Of particular interest to magnetic separation community are the reviews of the rare-earth permanent magnets. The value of these reviews is, however, reduced by the fact that both contributions were written in 1988 and do not cover recent, often quite dramatic developments in technological and market trends in rare-earth permanent magnets. 